\title{
Motion corrected sensitivity encoded isotropic projection reconstruction (SNIPR) for whole-heart coronary MRA
}

\author{
Jianing Pang ${ }^{1,2^{*}}$, Behzad Sharif ${ }^{1}$, Reza Arsanjani ${ }^{1}$, Louise E Thomson', John D Friedman', Daniel S Berman', \\ Debiao $\mathrm{Li}^{1,3}$ \\ From 16th Annual SCMR Scientific Sessions \\ San Francisco, CA, USA. 31 January - 3 February 2013
}

\section{Background}

As has been shown recently, the use of undersampled 3D projection reconstruction (3DPR) and image-based motion correction with $100 \%$ acquisition efficiency $[1,2]$ enables highly accelerated coronary MRA (CMRA) with high isotropic resolution. However, streaking artifact from undersampling significantly reduces the apparent signal-to-noise ratio (SNR). Moreover, affine motion correction distorts the $\mathrm{k}$-space trajectory, hence introducing more streaking. In this work, the SNIPR reconstruction [3], a self-calibrated sensitivity encoding scheme for 3DPR, is integrated with image-based motion correction to reduce streaking artifacts and thus improve image quality.

\section{Methods}

MR data was collected using an ECG-triggered, T2-prepared, fat-saturated bSSFP pulse sequence with 3DPR trajectory and a 12 channel receiver coil array $(\mathrm{TR} / \mathrm{TE}=3.2$ $\mathrm{ms} / 1.6 \mathrm{~ms}, \mathrm{FOV}=400 \mathrm{~mm}^{3}$, matrix size $=384^{3}$, flip angle $=90^{\circ}$, readout bandwidth $=900 \mathrm{~Hz} /$ pixel). Three datasets were collected from each subject in random order with 5800, 11500 or 20500 lines (imaging times: $2.8 \pm 0.3$, $5.6 \pm 0.6$ and $9.5 \pm 1.2 \mathrm{~min}$ ). Retrospective respiratory motion compensation was performed as previously described in [2]. The coil sensitivity maps were calculated with the motion corrected data using Walsh's method [4]. SNIPR extends the generalized SENSE framework described in [5] to 3D for iterative image reconstruction. Healthy volunteer scans $(\mathrm{N}=4)$ were performed successfully on a clinical 1.5T scanner (MAGNETOM Avanto, Siemens AG Healthcare, Erlangen, Germany) with IRB approval and

Biomedical Imaging Research Institute, Cedars Sinai Medical Center, Los Angeles, CA, USA

Full list of author information is available at the end of the article written consent. Offline reconstruction was implemented in MATLAB (MathWorks, Natick, MA). Image quality scores were assigned by an experienced reader blinded to the underlying techniques.

\section{Results}

The standard gridding shows significant streaking artifacts that increase with fewer lines, while SNIPR greatly reduces the amount of streaking in all datasets, improving the apparent SNR (Figure 1). Notably, the image qualities of 12000 and 20500 lines have no significant difference with SNIPR, although with 5800 lines there is a certain degree of blurring; this can be due to poor conditioning of the linear system, error in motion correction, and/or error in sensitivity maps. Table 1 shows the subjective image quality scores.

\section{Conclusions}

We have described a self-calibrated, motion-corrected 3D non-Cartesian sensitivity encoding reconstruction that greatly reduces streaking artifacts from undersampled 3DPR acquisitions, with isotropic $1 \mathrm{~mm}^{3}$ resolution and a 6 minutes scan time for whole-heart CMRA. Further studies are needed to characterize the noise performance of the proposed method. Also, the final image quality will benefit from improvements in motion correction and sensitivity map estimation, especially for highly undersampled datasets.

\section{Funding}

National Institute of Health grants nos. NIBIB EB002623 and NHLBI HL38698.AHA Postdoctoral Fellowship Award 11POST7390063.
C Biomed Central

C 2013 Pang et al; licensee BioMed Central Ltd. This is an Open Access article distributed under the terms of the Creative Commons Attribution License (http://creativecommons.org/licenses/by/2.0), which permits unrestricted use, distribution, and reproduction in any medium, provided the original work is properly cited. 


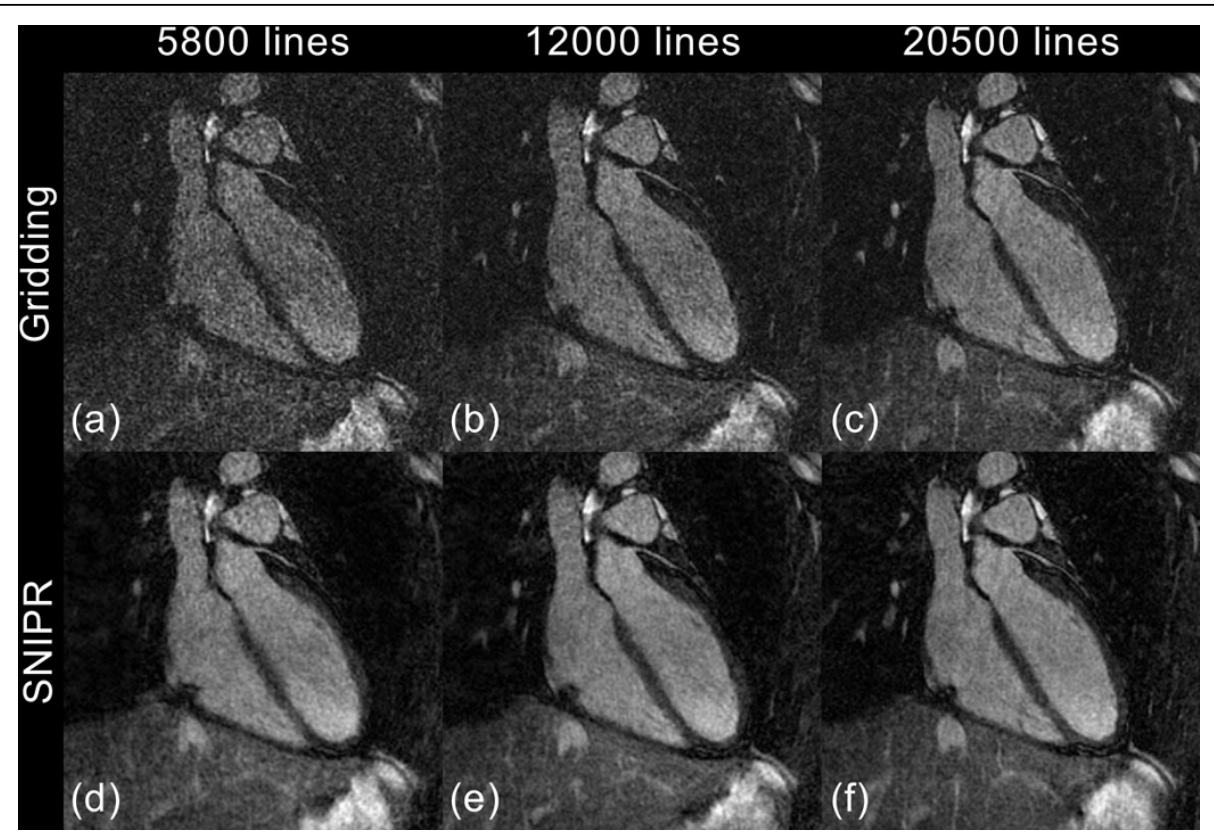

Figure 1 Comparing gridding and SNIPR. For various undersampling ratios, SNIPR significantly reduces streaking artifact, resulting in improved SNR.

Table 1 Image quality score results (range: $\mathbf{0 - 4}$ ) for different reconstruction methods and undersampling factors.

\begin{tabular}{ccccccc}
\hline & Gridding, 5800 lines & SNIPR, 5800 lines & Gridding, 12000 lines & SNIPR, 12000 lines & Gridding, 20500 lines & SNIPR, 20500 lines \\
\hline Score & $0.8 \pm 0.4$ & $1.1 \pm 0.2$ & $1.9 \pm 0.7$ & $2.5 \pm 0.4$ & $2.2 \pm 0.8$ & $2.5 \pm 0.7$ \\
\hline
\end{tabular}

\section{Author details}

${ }^{1}$ Biomedical Imaging Research Institute, Cedars Sinai Medical Center, Los Angeles, CA, USA. ${ }^{2}$ Radiology and Biomedical Engineering, Northwestern University, Chicago, IL, USA. ${ }^{3}$ Bioengineering, University of California, Los Angeles, CA, USA.

Published: 30 January 2013

\section{References}

1. Bhat:. MRM 2011, 65:1269.

2. Pang:. ISMRM 2012.

3. Pang:. ISMRM 2012.

4. Walsh:. MRM 2000, 43:682.

5. Pruessmann:. MRM 2001, 46:638.

doi:10.1186/1532-429X-15-S1-E65

Cite this article as: Pang et al:: Motion corrected sensitivity encoded isotropic projection reconstruction (SNIPR) for whole-heart coronary MRA. Journal of Cardiovascular Magnetic Resonance 2013 15(Suppl 1):E65.

Submit your next manuscript to BioMed Central and take full advantage of:

- Convenient online submission

- Thorough peer review

- No space constraints or color figure charges

- Immediate publication on acceptance

- Inclusion in PubMed, CAS, Scopus and Google Scholar

- Research which is freely available for redistribution 